Editorial

\title{
Special issue devoted to the 3rd World Conference on Physico- Chemical Methods in Drug Discovery and Development (PCMDDD-3)
}

\author{
Kin Tam ${ }^{1}$ and Zoran Mandić ${ }^{2}$ \\ ${ }^{1}$ Faculty of Health Science, University of Macau, Macau, China \\ E-mail: kintam@umac.mo; Tel.: +853-8397-8501; Fax: +853-8397-8500 \\ ${ }^{2}$ University of Zagreb, Faculty of Chemical Engineering and Technology, HR-10000, Zagreb, Croatia \\ E-mail: zmandic@fkit.hr; Tel: +38514597164
}

The present and following issues of ADMET and DMPK are dedicated to the 3rd World Conference on Physico-Chemical Methods in Drug Discovery and Development (PCMDDD-3) held in Dubrovnik, Croatia, 23-26 September 2013. PCMDDD-3 is organized as a biannual event and is intended to provide a place and common ground for the scientific community whose work is closely related to the application of physical chemistry in ADME and DMPK research to get together in an open and relaxing atmosphere to exchange ideas and discuss challenges. The topics of the last conference included: physico-chemical methods and instrumentation in the physico-chemical profiling of drug substances, ADME and DMPK, determination and characterization of different solid forms, hydrates and polymorphs, separation and analytical techniques of importance in medicinal chemistry and pharmaceutical research, computational methods and modelling. More details of the PCMDDD-3 can be found on the conference webpage: http://www.iapchem.org/page.php?page id=34.

Almost 150 delegates actively participated at the PCMDDD-3. Most of them presented their work either orally or through poster communication resulting in a diverse but well-balanced four-day programme.

These two issues review a small selection of about 15 papers representing a typical cross-section of the conference workings. The first part is composed of six papers. A review about multivariate analysis of hydrophobic parameters by Stefan Dove gives a historical overview and describes the importance of the multivariate approaches in the determination of hydrophobic descriptors. The author reviews his own work as well as the significant contributions of other authors in the field.

Three original scientific papers belong to the field of solubility of drug substances: the paper on the solubility of indomethacin was presented by the group led by John Comer and Alex Avdeef contributed two very interesting papers about the anomalous solubility behaviour of acidic and basic drugs.

The issue concludes with two papers dealing with drug formulations and their improvement. Radka Opatrilova and Josef Jampilek studied the effect of micro- and nano-ionized alaptide on the skin permeation of mupirocine. Rameshwar Deshmukh and Jitendra Naik presented their preliminary work on the preparation of diclofenac microparticles. 
We wish to thank all the authors of this and the following special issue for their high-quality papers. We would also like to thank the referees who critically evaluated the papers at short notice. Finally, we hope readers will enjoy the articles and find them interesting, useful and beneficial for their work. 